\title{
Study of backward terahertz radiation from intense picosecond laser-solid interactions using a multichannel calorimeter system
}

\author{
H. Liu ${ }^{1,6}$, G.-Q. Liao ${ }^{2}$, Y.-H. Zhang ${ }^{1,6}$, B.-J. Zhu ${ }^{1,6}$, Z. Zhang ${ }^{1}$, Y.-T. Li ${ }^{1,6,7}$, G. G. Scott ${ }^{3}$, D. Rusby ${ }^{3,4}$, \\ C. Armstrong ${ }^{3,4}$, E. Zemaityte ${ }^{3,4}$, P. Bradford ${ }^{5}$, N. Woolsey ${ }^{5}$, P. Huggard ${ }^{8}$, P. McKenna ${ }^{4}$, and D. Neely ${ }^{3,4}$ \\ ${ }^{1}$ Beijing National Laboratory for Condensed Matter Physics, Institute of Physics, Chinese Academy of Sciences, Beijing 100190, China \\ ${ }^{2}$ Key Laboratory for Laser Plasmas (MoE) and School of Physics and Astronomy, Shanghai Jiao Tong University, Shanghai 200240, \\ China \\ ${ }^{3}$ Central Laser Facility, Rutherford Appleton Laboratory, Didcot, Oxfordshire OX11 OQX, UK \\ ${ }^{4}$ Department of Physics, SUPA, University of Strathclyde, Glasgow G4 ONG, UK \\ ${ }^{5}$ Department of Physics, York Plasma Institute, University of York, Heslington, York YO10 5DD, UK \\ ${ }^{6}$ School of Physical Sciences, University of Chinese Academy of Sciences, Beijing 100049, China \\ ${ }^{7}$ Collaborative Innovation Center of IFSA (CICIFSA), Shanghai Jiao Tong University, Shanghai 200240, China \\ ${ }^{8}$ Space Science Department, STFC Rutherford Appleton Laboratory, Didcot OX11 OQX, UK \\ (Received 22 July 2018; revised 9 October 2018; accepted 13 November 2018)
}

\begin{abstract}
A multichannel calorimeter system is designed and constructed which is capable of delivering single-shot and broadband spectral measurement of terahertz $(\mathrm{THz})$ radiation generated in intense laser-plasma interactions. The generation mechanism of backward $\mathrm{THz}$ radiation (BTR) is studied by using the multichannel calorimeter system in an intense picosecond laser-solid interaction experiment. The dependence of the BTR energy and spectrum on laser energy, target thickness and pre-plasma scale length is obtained. These results indicate that coherent transition radiation is responsible for the low-frequency component $(<1 \mathrm{THz})$ of BTR. It is also observed that a large-scale pre-plasma primarily enhances the high-frequency component $(>3 \mathrm{THz})$ of BTR.
\end{abstract}

Keywords: multichannel calorimeter; backward terahertz radiation; generation mechanisms

\section{Introduction}

Intense terahertz $(\mathrm{THz})$ radiation sources have attracted increasing research interests due to their applications in coherent and incoherent control of matter, light and electron beam manipulation ${ }^{[1-4]}$. With terawatt and petawatt laser systems $^{[5]}$, $\mathrm{THz}$ radiation from high intensity laser-solid interactions has been demonstrated as a new intense $\mathrm{THz}$ source ${ }^{[6-11]}$. At present, the reported energy of intense lasersolid interaction-based $\mathrm{THz}$ sources ${ }^{[4,11]}$ has been at the same level as sources based on traditional accelerators ${ }^{[12]}$ and optical crystals ${ }^{[13]}$.

Terahertz radiation is generated from both target front and rear surfaces in intense laser-solid interaction ${ }^{[6,8]} . \mathrm{THz}$

Correspondence to: Y.-T. Li, Beijing National Laboratory of Condensed Matter Physics, Institute of Physics, Chinese Academy of Sciences, Beijing 100190, China; D. Neely, Central Laser Facility, STFC Rutherford Appleton Laboratory, Didcot OX110QX, UK. Email: ytli@iphy.ac.cn (Y.-T. Li); david.neely@stfc.ac.uk (D. Neely) radiation from target front surface propagates backward relative to the incident laser, which is referred as backward $\mathrm{THz}$ radiation (BTR) in this paper. And similarly, $\mathrm{THz}$ radiation from target rear surface is referred as forward $\mathrm{THz}$ radiation (FTR). There are mainly two mechanisms which account for FTR generation, including the target normal sheath acceleration radiation ${ }^{[10]}$ and coherent transition radiation ${ }^{[8]}$. However, the BTR generation mechanism still needs further clarification. A number of sources and processes such as fast electrons and plasma waves can produce electromagnetic pulses in intense laser-plasma interactions ${ }^{[6,7,14-16]}$. Several experiments have shown that pre-plasma scale length plays an important role in BTR generation $^{[6,7,17]}$. For pre-plasma with large scale length, $\mathrm{THz}$ radiation can be generated through the linear mode conversion (LMC) mechanism ${ }^{[14,18]}$. For pre-plasma with small scale length, the time-evolving directional surface fast electron currents (SFE) are thought to contribute to the 
$\mathrm{BTR}^{[19]}$. Sagisaka et al. proposed the antenna model which attributes the BTR to a fast radial current confined within the target surface ${ }^{[20]}$. Coherent transition radiation (CTR) by laser-produced currents at the target front surface is also proposed as an effective mechanism for BTR generation ${ }^{[15]}$. However, no crucial experimental evidence has been found so far. It should be noted that most of the above mechanisms are demonstrated in intense femtosecond laser-solid interactions experiments. Therefore, these mechanisms may not apply to BTR generated in interactions between high-energy picosecond laser pulse and solid targets.

It has been found that the high-frequency component $(>10 \mathrm{THz}$ ) is dominant in backward $\mathrm{THz}$ radiation due to $\mathrm{LMC}^{[7]}$. However, the CTR or SFE mainly accounts for the BTR at low frequencies $(<3 \mathrm{THz})^{[15,19]}$. Therefore, spectral measurement of the emitted $\mathrm{THz}$ wave is necessary to determine the BTR generation mechanism. However, the existing $\mathrm{THz}$ spectrum measurement technology is not ideally applicable for $\mathrm{THz}$ sources based on intense laserplasma interactions (ILPI). This is because the repetition rate of many such sources is quite low at present, typically below $1 \mathrm{~Hz}$. Also, the fluctuations between shots can be large in real experiments. As a result, multi-shot scanning-based methods are quite inefficient and potentially inaccurate when used to characterize ILPI-based sources, such as electrooptic sampling $^{[21]}$ and autocorrelation measurement based on a Michelson interferometer ${ }^{[22]}$. Moreover, the working bandwidth of general electro-optic crystals also limits the application of electro-optic sampling techniques. The bandwidth of BTR generated in intense laser-plasma interactions can reach tens of $\mathrm{THz}^{[6,7]}$. However, the effective bandwidth is only $5.3 \mathrm{THz}$ for $\mathrm{ZnTe}$ and $7 \mathrm{THz}$ for $\mathrm{GaP}^{[23]}$. In addition, the existing frequency-domain methods also share this disadvantage such as THz spectrometers based on prism ${ }^{[24]}$ and consecutive dispersion grating ${ }^{[25]}$.

In this paper, an eight-channel calorimeter system has been designed and constructed to deliver single-shot spectral measurements of BTR generated in an intense picosecond lasersolid interaction experiment. The system has components including pyroelectric detectors, high resistance float zone silicon (HRFZ-Si) $\mathrm{THz}$ beam splitters and $\mathrm{THz}$ band pass filters. In the experiment, the driving laser energy, target thickness and pre-pulse condition are varied. It is found that the transition radiation by refluxing fast electrons contributes greatly to the low-frequency component $(<1 \mathrm{THz})$ of BTR. The large-scale pre-plasma plays an important role in the generation of the high-frequency component $(>3 \mathrm{THz})$.

\section{Design and calibration of multichannel calorimeter system}

A multichannel calorimeter system consisting of pyroelectric detectors has been used to obtain spectral information of

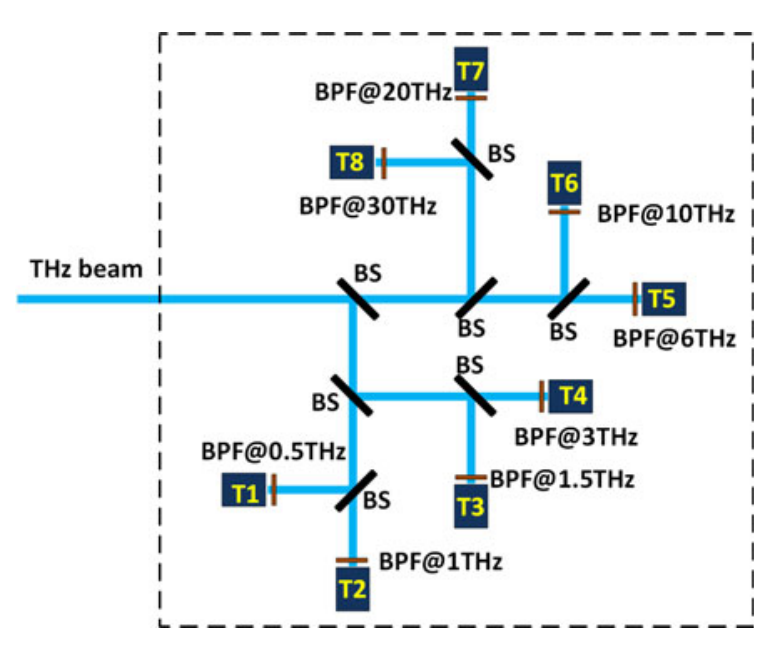

Figure 1. Schematic layout of eight-channel $\mathrm{THz}$ calorimeter system.

broad-band plasma-based THz sources in one laser shot ${ }^{[26]}$. Coherent $\mathrm{THz}$ radiation in two filtered bands was measured to the bunch length in Tsinghua Thomson scattering X-ray source $^{[27]}$. In this paper, the calorimeter system has eight channels and each channel contains an HRFZ-Si THz beam splitter, a THz band pass filter and a pyroelectric detector, as shown in Figure 1. The incoming THz beam is divided and guided into each channel by seven HRFZ-Si THz beam splitters. In each channel, a THz band pass filter is set in front of the pyroelectric detector. As a result, the calorimeter system can sample the $\mathrm{THz}$ radiation in eight different bands in a single laser shot.

The flat transmission curves of the HRFZ-Si beam splitter and responsivity of the pyroelectric detector enable the calorimeter system to have a quite broad sensitivity in the $\mathrm{THz}$ band. HRFZ-Si is an isotropic crystalline material which is suitable to serve as a $\mathrm{THz}$ beam splitter material ${ }^{[28]}$. In the calorimeter, $1 \mathrm{~mm}$-thick HRFZ-Si wafers with both sides polished are used as the single-pass $\mathrm{THz}$ beam splitters. The transmission of $1 \mathrm{~mm}$-thick HRFZ-Si wafer was calibrated using a Fourier transform infrared (FTIR) spectrometer in the previous work ${ }^{[6-8]}$. According to the calibration results, it maintains around 54\% transmission from 1.2 to $1000 \mu \mathrm{m}$. The pyroelectric detector in our calorimeter system consists of a single lithium tantalite $\left(\mathrm{LiTaO}_{3}\right)$ sensor of surface area $4 \mathrm{~mm}^{2}$, which has a broad spectral response from 0.1 to $1000 \mu \mathrm{m}^{[29]}$. The original signal from the sensor is coupled to an amplifying and readout circuit.

The sensitivity of the pyroelectric detector in the nearinfrared band is calibrated with a Ti:sapphire laser, which outputs $100 \mathrm{fs}$ pulses at $10 \mathrm{~Hz}$ with a wavelength centered around $800 \mathrm{~nm}$. The energy of the laser pulse incident onto the sensor of the detector is varied and measured by a calibrated power meter. As shown in Figure 2, there is a high linearity between the output signal and the input laser energy, with a sensitivity of the detector being 


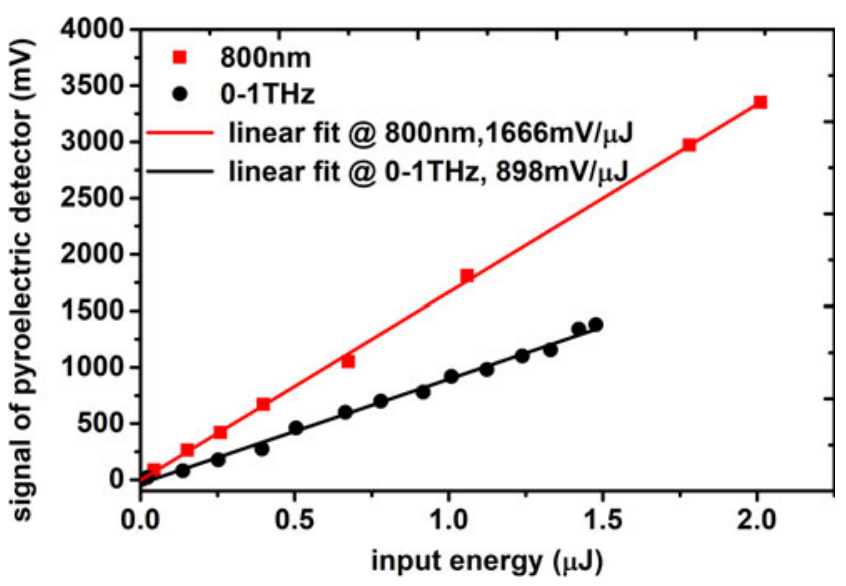

Figure 2. Linear response of the detector to energy of near-infrared and $\mathrm{THz}$ wavelengths.

$1666 \pm 10 \mathrm{mV} / \mu \mathrm{J}$ for the $800 \mathrm{~nm}$ laser. The detector's sensitivity is also calibrated in the $\mathrm{THz}$ band. $\mathrm{THz}$ pulses are emitted from a lithium niobate $\left(\mathrm{LiNbO}_{3}\right)$ crystal when pumped with $800 \mathrm{~nm}$ pulse-front-tilted laser ${ }^{[2]}$. The spectrum of emitted THz waves is broad from 0.1 to $1 \mathrm{THz}$ as measured with an electro-optic sampler. The energy of emitted $\mathrm{THz}$ pulses is measured using a calibrated pyroelectric detector $^{[30]}$, whose sensitivity is $25 \pm 0.875 \mathrm{mV} / \mu \mathrm{J}$. With cross calibration, the sensitivity of our detector at $0.1-1 \mathrm{THz}$ is measured to be $898 \pm 11 \mathrm{mV} / \mu \mathrm{J}$. A ratio of sensitivity in near-infrared and $\mathrm{THz}$ band of 1.86 is calculated. The relatively low sensitivity of the pyroelectric detector in the $\mathrm{THz}$ band is probably due to the decreased efficiency of power absorption in the coating on the pyroelectric crystal. Huggard et al. [31] show that the coating can have an increased reflectivity in the far-infrared and $\mathrm{THz}$ band.

The band pass filters used in the calorimeter are fabricated from gold-mesh frequency-selective surfaces ${ }^{[32]}$. Eight filters were installed with center frequencies equal to $0.5 \mathrm{THz}, 1 \mathrm{THz}, 1.5 \mathrm{THz}, 3 \mathrm{THz}, 6 \mathrm{THz}, 10 \mathrm{THz}, 20 \mathrm{THz}$ and $30 \mathrm{THz}$, respectively. Each filter has its own working band with peak transmission above $70 \%$ at the center wavelength which has also been calibrated using FTIR spectrometer. The eight filters share negligible transmission in the overlapping regions between each other.

\section{BTR measurements in an intense picosecond laser- solid interaction experiment}

With all components ready, the eight-channel calorimeter system is constructed as shown in Figure 3(a). An eightchannel power supply is made for the pyroelectric detectors. The signals from the detectors are recorded by two $500 \mathrm{MHz}$ oscilloscopes. The eight-channel calorimeter system is used in an intense laser-solid interaction experiment to characterize the $\mathrm{THz}$ radiation spectrum from the target front surface. The experiment is performed at the Vulcan laser facility ${ }^{[33]}$ of the Rutherford Appleton Laboratory, UK. A $1053 \mathrm{~nm}$ laser pulse with energy up to $100 \mathrm{~J}$ and duration 1 ps is best focused onto copper foil targets at an intensity of $10^{20} \mathrm{~W} / \mathrm{cm}^{2}$ at an incident angle of $30^{\circ}$, as shown in Figure 3(a). $\mathrm{THz}$ emission generated from the front surface of the target is collected by a $50 \mathrm{~mm}$ diameter TPX lens placed at an angle of $60^{\circ}$ from the target normal direction. The beam is reduced to $25 \mathrm{~mm}$ diameter using two off-axis parabolic mirrors with focal length $200 \mathrm{~mm}$ and $100 \mathrm{~mm}$, respectively. The reduced beam is guided into the eight-channel calorimeter through a $50 \mathrm{~mm}$ TPX window. The transmission of the TPX lens and window is also measured with the FTIR spectrometer. In one of the eight channels, none filter was added so that the total energy of the $\mathrm{THz}$ radiation could be monitored. The remaining seven channels are equipped with band pass filters whose central frequencies are $0.5 \mathrm{THz}, 1 \mathrm{THz}, 1.5 \mathrm{THz}, 3 \mathrm{THz}$, $6 \mathrm{THz}, 10 \mathrm{THz}$ and $20 \mathrm{THz}$, respectively. To avoid any disturbance of the calorimeters by pickup of electromagnetic pulses $^{[34,35]}$ and high-energy ionizing radiation such as $\mathrm{X}$-rays from the laser-plasma interaction, the calorimeter system is well shielded with a metal box and lead sheets.

The spectral sensitivity of the system is necessary to obtain the $\mathrm{THz}$ spectrum from the measured signals. The spectral sensitivity mainly depends on the transmission properties of the $\mathrm{THz}$ optics used in the system and the spectral responsivity of the pyroelectric detector. Each band pass filter has a narrow passing band. In the narrow band, the transmissions of the band pass filter, HRFZ-Si beam splitter, TPX window and lens, as well as the spectral sensitivity of the pyroelectric detector, are simplified to be constant. Considering all the factors, the spectral sensitivity of the multichannel calorimeter system $S\left(f_{i}\right)$ can be written as

$$
\begin{aligned}
S\left(f_{i}\right)= & T_{\text {lens }}\left(f_{i}\right) \cdot T_{\text {window }}\left(f_{i}\right) \cdot E_{i} \\
& \cdot T_{\mathrm{BPF}}\left(f_{i}\right) \cdot S_{\text {detector }}\left(f_{i}\right) .
\end{aligned}
$$

Here, $i$ is the channel number from 1 to $8, f_{i}$ is the central frequency of band pass filter located in the $i$ th channel, $T_{\text {lens }}\left(f_{i}\right)$ and $T_{\text {window }}\left(f_{i}\right)$ are the transmissions of the TPX lens and window at $f_{i}$, respectively, $E_{i}$ is the relative energy portion allocated to the $i$ th channel determined by the layout of HRFZ-Si beam splitters, $T_{\mathrm{BPF}}\left(f_{i}\right)$ is the transmission of the band pass filter at $f_{i}$, and $S_{\text {detector }}\left(f_{i}\right)$ is the relative sensitivity of the detector at $f_{i}$. Table 1 lists the factors for the system spectral sensitivity calculation which is based on the calibration of the $\mathrm{THz}$ detectors and other components. With the system spectral sensitivity and measured signals, the THz radiation spectral intensity $I\left(f_{i}\right)$ is inversed as

$$
I\left(f_{i}\right)=V_{i} /\left[E_{\text {laser }} \cdot S\left(f_{i}\right) \cdot W_{i}\right] .
$$


(a)

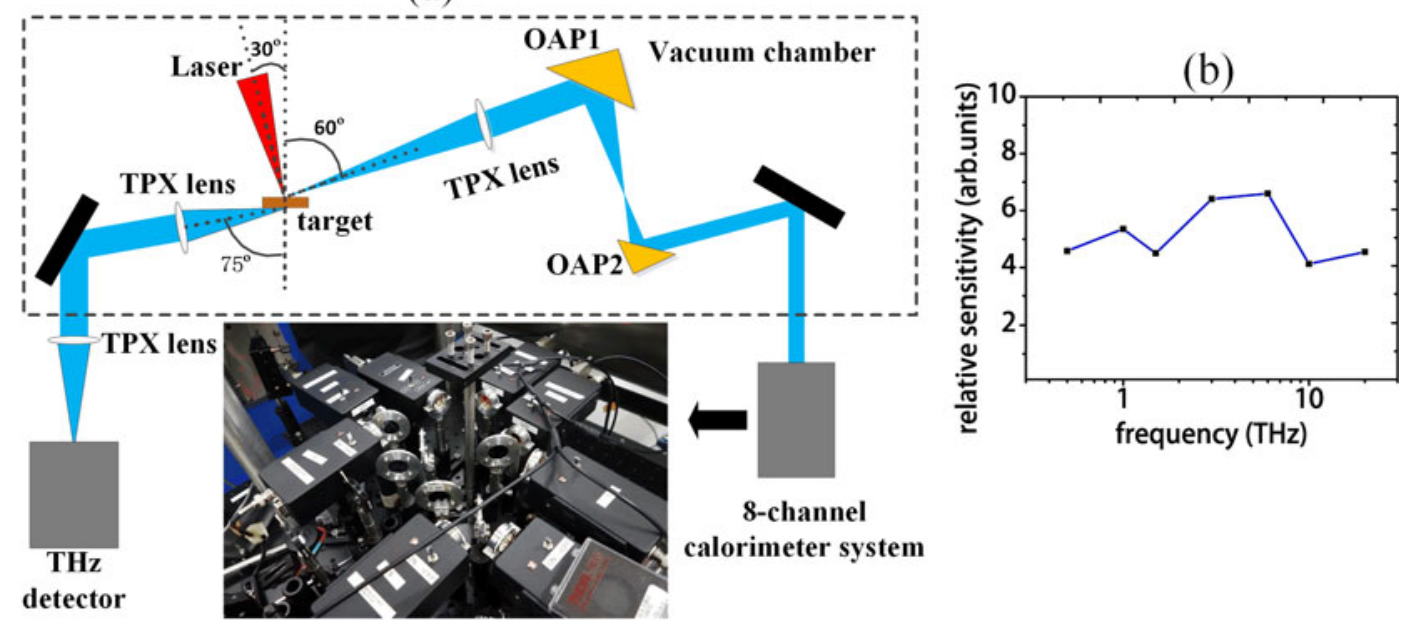

Figure 3. (a) Experimental setup of THz radiation spectrum measurement in ultra-intense laser-plasma interaction experiment. (b) Spectral sensitivity of the multichannel calorimeter system.

Table 1. Factors for the spectral sensitivity of the multichannel system.

\begin{tabular}{ccccccc}
\hline$i$ & $f_{i} / \mathrm{THz}$ & $T_{\text {lens }}\left(f_{i}\right)$ & $T_{\text {window }}\left(f_{i}\right)$ & $E_{i}$ & $T_{\mathrm{BPF}}\left(f_{i}\right)$ & $S_{\text {detector }}\left(f_{i}\right)$ \\
\hline 1 & 0.5 & 0.8 & 0.8 & 0.114 & 0.95 & 0.66 \\
2 & 1 & 0.8 & 0.8 & 0.134 & 0.78 & 0.80 \\
3 & 1.5 & 0.8 & 0.8 & 0.097 & 0.85 & 0.85 \\
4 & 3 & 0.81 & 0.81 & 0.134 & 0.80 & 0.91 \\
5 & 6 & 0.7 & 0.7 & 0.157 & 0.90 & 0.95 \\
6 & 10 & 0.6 & 0.6 & 0.134 & 0.90 & 0.95 \\
7 & 20 & 0.69 & 0.69 & 0.114 & 0.88 & 0.95 \\
8 & - & - & - & 0.116 & - & - \\
\hline
\end{tabular}

Here, the $E_{\text {laser }}$ is shot energy of the intense laser which is used to normalize the spectrum to reduce the effects of shotto-shot fluctuations. $W_{i}$ is the bandwidth of the pass filter in the $i$ th channel.

Figure 3(b) shows the calculated spectral sensitivity of the calorimeter system. The system has a broad sensitivity from 0.5 to $20 \mathrm{THz}$, which is advantageous for spectral measurement of the $\mathrm{THz}$ radiation. Combining all the factors, the system has relatively high sensitivity at $3 \mathrm{THz}$ and $6 \mathrm{THz}$. The low sensitivity at $0.5 \mathrm{THz}$ is mainly due to the low responsivity of the pyroelectric detector to lowfrequency part. And the system is less sensitive at high frequencies such as $10 \mathrm{THz}$ and $20 \mathrm{THz}$ because of the absorption of TPX material. There are several factors which may cause inaccuracy of the $\mathrm{THz}$ spectrum measurement using the system. The fluctuations of shot spots could lead to a direction instability of $\mathrm{THz}$ beam. This will lead to the deviation of a portion of the original $\mathrm{THz}$ beam into each channel. This deviation can be reduced by applying a collimating lens with longer focal length or decreasing the distance between the instrument and the target. The use of TPX lens and window causes huge absorption for frequencies large than $10 \mathrm{THz}$, which would modify the original spectrum. It is preferred to utilize materials with flat transmission such as HRFZ-Si or reflection-style optics like off-axis parabola mirror. In addition, other factors like the diffraction and air absorption loss also affect the system spectral sensitivity. Careful calibration for these factors contributes to highly accurate system sensitivity.

\section{Experimental results}

Figure 4(a) shows the dependence of the BTR energy from a $5 \mu \mathrm{m}$ copper target driven with a laser energy varying from 0 to $54 \mathrm{~J}$. The BTR energy increases linearly as the driven energy increases. Figure 4(b) shows the measured BTR spectrum using the multichannel calorimeter system under different pump laser energy conditions. The spectrum of BTR is quite broad and the low-frequency component $(<3 \mathrm{THz})$ has a relatively high spectral intensity. This helps us to conclude that the low-frequency component is laser intensity-dependent, especially those lower than $1 \mathrm{THz}$. The higher driven energy means higher focal intensity on the target surface, which enhances the fast electrons contributing to terahertz radiation. But the spectral intensity of the highfrequency component changes little, indicating the high frequency $\mathrm{THz}$ radiation is not sensitive to the laser intensity. This is an evidence that the generation mechanism of the low- and high-frequency components is different.

The dependence of BTR energy and spectrum on the thickness of copper foil target is shown in Figures 5(a) and 5(b). The intensity of low-frequency components increases as the target thickness rises from $1 \mu \mathrm{m}$ to $20 \mu \mathrm{m}$. As the thickness is further increased to $100 \mu \mathrm{m}$, the low-frequency component $(<1 \mathrm{THz})$ decreases significantly. The total BTR energy shows a similar dependence on target thickness. 

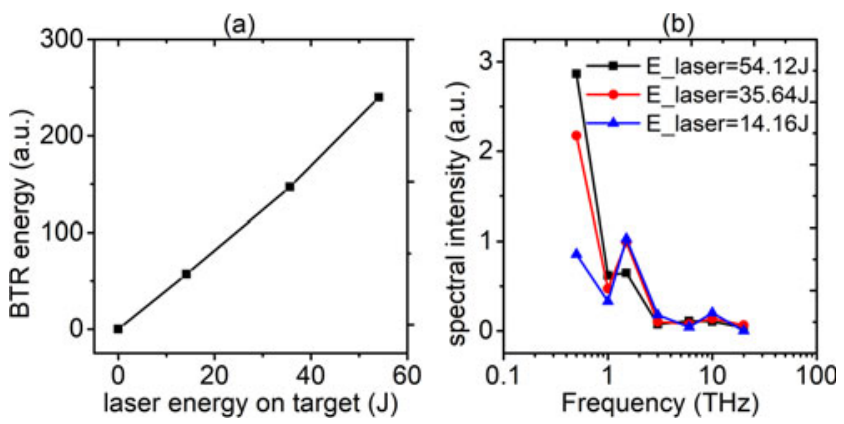

Figure 4. (a) The measured BTR energy from a $5 \mu \mathrm{m}$ copper target as a function of the pump laser energy. (b) The measured BTR spectrum with driven laser energy on target varying from $14 \mathrm{~J}$ to $54 \mathrm{~J}$ from a $5 \mu \mathrm{m}$ copper target.
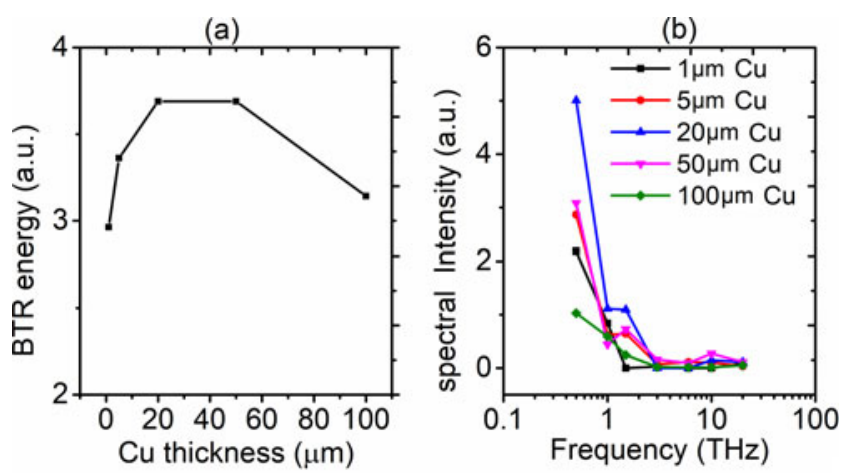

Figure 5. (a) The measured BTR energy as a function of copper foil target thickness. (b) The measured BTR spectrum with copper foil target thickness varying from $1 \mu \mathrm{m}$ to $100 \mu \mathrm{m}$.

The effect of pre-plasma on the BTR generation is also studied during our experiment. A $1 \%$ portion of the main laser beam is extracted and co-linearly injected as a pre-pulse with a variable delay between the pre-pulse and main pulse. The pre-pulse will generate pre-plasma and the plasma will expand during the delay time. With longer delay between pre-pulse and main pulse, pre-plasma with larger scale length is formed. The dependence of BTR energy on the delay is shown as Figure 6(a). The BTR energy increases as the pre-plasma scale length grows. The BTR spectrum with different delays is as shown in Figure 6(b). When the delay increases from 0 to $400 \mathrm{ps}$, more BTR of low frequency is generated. When the delay further increases to $1000 \mathrm{ps}$, the interesting thing is that the intensity of the low-frequency component $(<1 \mathrm{THz})$ decreases slightly and the higherfrequency component $(>3 \mathrm{THz})$ is enhanced.

\section{Discussion}

The dominant component of backward terahertz radiation in our experiment is lower than $1 \mathrm{THz}$ in frequency, which can be interpreted with the coherent transition radiation mechanism. According to the CTR theory, the wavelength
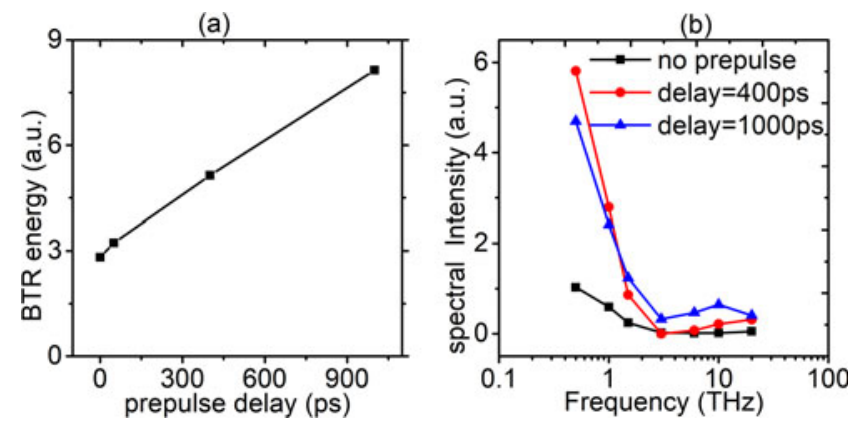

Figure 6. (a) The measured BTR energy from a $100 \mu \mathrm{m}$ copper target as a function of delay between pre-pulse and main pulse. (b) The measured BTR spectrum from a $100 \mu \mathrm{m}$ copper target with different delays between pre-pulse and main pulse, no pre-pulse, 400 ps and 1000 ps, respectively.

$\lambda$ of coherent $\mathrm{THz}$ radiation is longer than the longitudinal length of electron bunches $\sigma_{e z}$. In our case, $\sigma_{e z}$ is estimated to be $c \cdot \tau_{L} \sim 300 \mu \mathrm{m}$, where $\tau_{L}$ is the laser pulse length ${ }^{[8]}$. As the driving laser energy increases, the number of fast electrons increases, which enhances the CTR ${ }^{[36]}$. PIC simulation has shown that both the laser-accelerated backward electron bunches and refluxing electrons contribute to the BTR generated through $\mathrm{CTR}^{[15]}$. The laser-produced electron bunches account for the primary $\mathrm{THz}$ pulses. In intense laser-solid interactions, some of laser-accelerated electrons are recirculated inside the target by the sheath field which exists both on the target front and rear surfaces ${ }^{[37]}$. And subsequent $\mathrm{THz}$ pulses are emitted when these refluxing electrons cross the vacuum-target interfaces.

The observed dependence of backward $\mathrm{THz}$ energy on the target thickness suggests the CTR by the refluxing electrons contributes to the BTR in our experiment. It has been found that the target thickness has a significant effect on the recirculation of fast electrons inside the conductive foil. As the target thickness increases, the time to complete a pass through the target will gradually increase and the total number of recirculations will decrease ${ }^{[37,38]}$. In our experiment, the $20 \mu \mathrm{m}$ thick copper target is optimum for BTR generation. The pre-pulse will ionize the target rear surface and affect the formation of sheath field when thin targets are used. This reduces the reflection effect of the sheath field. As a result, less BTR is measured with $1 \mu \mathrm{m}$ and $5 \mu \mathrm{m}$ thick targets. For thicker targets such as $50 \mu \mathrm{m}$ and $100 \mu \mathrm{m}$, the decrease of BTR energy is due to the decrease of the number of transits of the refluxing fast electrons across the target.

Other mechanisms such as surface fast electron currents and LMC cannot account for the dependence of BTR on target thickness. In these models, only sources like currents and plasma waves related to the pre-plasma are considered. As the target gets thicker, BTR should not change because of the relatively constant pre-plasma condition according to the surface fast electron currents and LMC model. However, the energy of the BTR decreases a lot with the $100 \mu \mathrm{m}$ foil 
target, especially the low-frequency component. In addition, $\mathrm{THz}$ radiation by $\mathrm{LMC}$ mechanism features a high-frequency $(>10 \mathrm{THz})$ component which disagrees with the measured BTR spectrum.

However, the LMC mechanism will become important as pre-plasma scale length increases. With a large-scale pre-plasma, laser absorption efficiency will be significantly improved by laser self-focusing and filament effects ${ }^{[39,40]}$. In our experiment, the low-frequency component is significantly enhanced when a pre-pulse is added 400 ps ahead of the main pulse. This is attributed to the increase of the number of fast electrons due to improved laser absorption efficiency. When the scale length of pre-plasma further increases, the enhanced CTR by more fast electrons cannot come out of the large-scale plasma. However, large-scale pre-plasma is helpful for $\mathrm{LMC}^{[7]}$. And this is why the low-frequency component $(<1 \mathrm{THz})$ is weakened and highfrequency component $(>3 \mathrm{THz})$ is enhanced when the delay is further increased to $1000 \mathrm{ps}$. However, considering the contribution of the high-frequency component $(>3 \mathrm{THz})$, the total energy of BTR still increases. This phenomenon indicates that the LMC mechanism is starting to make a measurable contribution under this condition.

\section{Conclusion}

A simple multichannel calorimeter system is designed and constructed, which consists of HRFZ-Si THz beam splitters, $\mathrm{THz}$ band pass filters and pyroelectric detectors. It provides a convenient single-shot solution to characterize the spectrum of broad-band $\mathrm{THz}$ radiation generated in an intense laserplasma interaction. Spectral measurements of backward $\mathrm{THz}$ radiation are performed using the multichannel calorimeter system in an intense picosecond laser-solid interaction experiment. The low-frequency component $(<1 \mathrm{THz})$ is dominant for the BTR measured in the experiment. The BTR is attributed to coherent transition radiation by laseraccelerated fast electrons as they cross the plasma-vacuum surface. The dependence of BTR energy and spectrum on the target thickness indicates that CTR by refluxing fast electrons contributes to the BTR. The LMC mechanism starts to work with a large-scale pre-plasma, which enhances the intensity of the high-frequency components $(>3 \mathrm{THz})$ of the signal.

\section{Acknowledgements}

This work is supported by the Newton China-UK joint research grant on laser-ion acceleration and novel terahertz radiation, and EPSRC grant EP/K022415/1 on advanced laser-ion acceleration strategies toward next generation healthcare and EPSRC grant EP/R006202/1. This work is also supported by the National Natural
Science Foundation of China (Nos. 11520101003 and 11861121001), the Strategic Priority Research Program of the Chinese Academy of Sciences (Nos. XDB16010200 and XDB07030300). G.Q.L. would like to acknowledge support from the National Postdoctoral Program for Innovative Talents (No. BX201600106). Data associated with research published in this paper can be accessed at: http://dx.doi.org/ 10.5286/edata/715.

\section{References}

1. T. Kampfrath, K. Tanaka, and K. A. Nelson, Nat. Photon. 7, 680 (2013)

2. H. A. Hafez, X. Chai, A. Ibrahim, S. Mondal, D. Férachou, X. Ropagnol, and T. Ozaki, J. Opt. 18, 093004 (2016).

3. E. A. Nanni, W. R. Huang, K. H. Hong, K. Ravi, A. Fallahi, G. Moriena, R. J. Miller, and F. X. Kartner, Nature Commun. 6, 8486 (2015).

4. D. R. Symes, D. Neely, J. L. Collier, D. A. Jaroszynski, and D. Dumitras, AIP Conf. Proc. 1228, 444 (2010).

5. C. Danson, D. Hillier, N. Hopps, and D. Neely, High Power Laser Sci. Eng. 3, e3 (2015).

6. C. Li, G. Q. Liao, M. L. Zhou, F. Du, J. L. Ma, Y. T. Li, W. M. Wang, Z. M. Sheng, L. M. Chen, and J. Zhang, Opt. Express 24, 4010 (2016).

7. G. Q. Liao, Y. T. Li, C. Li, L. N. Su, Y. Zheng, M. Liu, W. M. Wang, Z. D. Hu, W. C. Yan, J. Dunn, J. Nilsen, J. Hunter, Y. Liu, X. Wang, L. M. Chen, J. L. Ma, X. Lu, Z. Jin, R. Kodama, Z. M. Sheng, and J. Zhang, Phys. Rev. Lett. 114, $255001(2015)$.

8. G. Q. Liao, Y. T. Li, Y. H. Zhang, H. Liu, X. L. Ge, S. Yang, W. Q. Wei, X. H. Yuan, Y. Q. Deng, B. J. Zhu, Z. Zhang, W. M. Wang, Z. M. Sheng, L. M. Chen, X. Lu, J. L. Ma, X. Wang, and J. Zhang, Phys. Rev. Lett. 116, 205003 (2016).

9. G. Q. Liao, Y. T. Li, C. Li, H. Liu, Y. H. Zhang, W. M. Jiang, X. H. Yuan, J. Nilsen, T. Ozaki, W. M. Wang, Z. M. Sheng, D. Neely, P. McKenna, and J. Zhang, Plasma Phys. Control. Fusion 59, 014039 (2017).

10. A. Gopal, S. Herzer, A. Schmidt, P. Singh, A. Reinhard, W. Ziegler, D. Brommel, A. Karmakar, P. Gibbon, U. Dillner, T. May, H. G. Meyer, and G. G. Paulus, Phys. Rev. Lett. 111, 074802 (2013).

11. A. Gopal, P. Singh, S. Herzer, A. Reinhard, A. Schmidt, U. Dillner, T. May, H. G. Meyer, W. Ziegler, and G. G. Paulus, Opt. Lett. 38, 4705 (2013).

12. Z. Wu, A. S. Fisher, J. Goodfellow, M. Fuchs, D. Daranciang, M. Hogan, H. Loos, and A. Lindenberg, Rev. Sci. Instrum. 84, 022701 (2013).

13. C. Vicario, A. V. Ovchinnikov, S. I. Ashitkov, M. B. Agranat, V. E. Fortov, and C. P. Hauri, Opt. Lett. 39, 6632 (2014).

14. Z. M. Sheng, K. Mima, J. Zhang, and H. Sanuki, Phys. Rev. Lett. 94, 095003 (2005).

15. W. J. Ding, Z. M. Sheng, and W. S. Koh, Appl. Phys. Lett. 103, 204107 (2013).

16. H. Hamster, A. Sullivan, S. Gordon, W. White, and R. W. Falcone, Phys. Rev. Lett. 71, 2725 (1993).

17. C. Li, M. L. Zhou, W. J. Ding, F. Du, F. Liu, Y. T. Li, W. M. Wang, Z. M. Sheng, J. L. Ma, L. M. Chen, X. Lu, Q. L. Dong, Z. H. Wang, Z. Lou, S. C. Shi, Z. Y. Wei, and J. Zhang, Phys. Rev. E 84, 036405 (2011).

18. Z.-M. Sheng, K. Mima, and J. Zhang, Phys. Plasmas 12, $123103(2005)$ 
19. Y. T. Li, C. Li, M. L. Zhou, W. M. Wang, F. Du, W. J. Ding, X. X. Lin, F. Liu, Z. M. Sheng, X. Y. Peng, L. M. Chen, J. L. Ma, X. Lu, Z. H. Wang, Z. Y. Wei, and J. Zhang, Appl. Phys. Lett. 100, 254101 (2012).

20. A. Sagisaka, H. Daido, S. Nashima, S. Orimo, K. Ogura, M. Mori, A. Yogo, J. Ma, I. Daito, A. S. Pirozhkov, S. V. Bulanov, T. Z. Esirkepov, K. Shimizu, and M. Hosoda, Appl. Phys. B 90, 373 (2008).

21. Q. Wu and X. C. Zhang, Appl. Phys. Lett. 67, 3523 (1995).

22. L.-L. Zhang, W.-M. Wang, T. Wu, R. Zhang, S.-J. Zhang, C.L. Zhang, Y. Zhang, Z.-M. Sheng, and X.-C. Zhang, Phys. Rev. Lett. 119, 235001 (2017).

23. Q. Wu and X. C. Zhang, Appl. Phys. Lett. 70, 1784 (1997).

24. A. S. Meijer, J. J. H. Pijpers, H. K. Nienhuys, M. Bonn, and W. J. van der Zande, J. Opt. A 10, 095303 (2008).

25. S. Wesch, B. Schmidt, C. Behrens, H. Delsim-Hashemi, and P. Schmüser, Nucl. Instrum. Methods Phys. Res. A 665, 40 (2011).

26. C. Li, G.-Q. Liao, and Y.-T. Li, High Power Laser Sci. Eng. 3, e15 (2015).

27. X. Su, L. Yan, Y. Du, Z. Zhang, Z. Zhou, D. Wang, L. Zheng, Q. Tian, W. Huang, and C. Tang, Nucl. Instrum. Methods Phys. Res. B 402, 157 (2017).

28. http://www.tydexoptics.com/pdf/THz_Materials.pdf.

29. http://www.eltecinstruments.com/PDF/Ds/Data\%20Sheet $\% 20-\% 20$ Model\%20400.pdf.

30. X.-J. Wu, J.-L. Ma, B.-L. Zhang, S.-S. Chai, Z.-J. Fang, C.Y. Xia, D.-Y. Kong, J.-G. Wang, H. Liu, C.-Q. Zhu, X. Wang, C.-J. Ruan, and Y.-T. Li, Opt. Express 26, 7107 (2018).

31. P. G. Huggard, S. Zeuner, K. Goller, H. Lengfellner, and W. Prettl, J. Appl. Phys. 75, 616 (1994).

32. http://www.thorlabschina.cn/newgrouppage9.cfm? objectgroup_id $=7611$.
33. C. N. Danson, J. Collier, D. Neely, L. J. Barzanti, A. Damerell, C. B. Edwards, M. H. R. Hutchinson, M. H. Key, P. A. Norreys, and D. A. Pepler, Opt. Acta Int. J. Opt. 45, 1653 (1998).

34. M. J. Mead, D. Neely, J. Gauoin, R. Heathcote, and P. Patel, Rev. Sci. Instrum. 75, 4225 (2004).

35. P. Bradford, N. C. Woolsey, G. G. Scott, G. Liao, H. Liu, Y. Zhang, B. Zhu, C. Armstrong, S. Astbury, C. Brenner, P. Brummitt, F. Consoli, I. East, R. Gray, D. Haddock, P. Huggard, P. J. R. Jones, E. Montgomery, I. Musgrave, P. Oliveira, D. R. Rusby, C. Spindloe, B. Summers, E. Zemaityte, Z. Zhang, Y. Li, P. McKenna, and D. Neely, High Power Laser Sci. Eng. 6, e21 (2018).

36. C. B. Schroeder, E. Esarey, J. Van Tilborg, and W. P. Leemans, Phys. Rev. E 69, 016501 (2004).

37. M. N. Quinn, X. H. Yuan, X. X. Lin, D. C. Carroll, O. Tresca, R. J. Gray, M. Coury, C. Li, Y. T. Li, C. M. Brenner, A. P. L. Robinson, D. Neely, B. Zielbauer, B. Aurand, J. Fils, T. Kuehl, and P. McKenna, Plasma Phys. Control. Fusion 53, 025007 (2011).

38. Y. Sentoku, T. E. Cowan, A. Kemp, and H. Ruhl, Phys. Plasmas 10, 2009 (2003).

39. P. McKenna, D. C. Carroll, O. Lundh, F. Nürnberg, K. Markey, S. Bandyopadhyay, D. Batani, R. G. Evans, R. Jafer, S. Kar, D. Neely, D. Pepler, M. N. Quinn, R. Redaelli, M. Roth, C. G. Wahlström, X. H. Yuan, and M. Zepf, Laser Part Beams 26, 591 (2008).

40. T. Z. Esirkepov, J. K. Koga, A. Sunahara, T. Morita, M. Nishikino, K. Kageyama, H. Nagatomo, K. Nishihara, A. Sagisaka, H. Kotaki, T. Nakamura, Y. Fukuda, H. Okada, A. S. Pirozhkov, A. Yogo, M. Nishiuchi, H. Kiriyama, K. Kondo, M. Kando, and S. V. Bulanov, Nucl. Instrum. Methods Phys. Res. A 745, 150 (2014). 